$46^{\circ}$ C. Arnold, Spec. Hew. ron zu suboutanem Gebrauch verwendeten Medicamentex.

\title{
Ueber das specifische Gewicht von zu subcutanem Gebrauch verwendeten Medicamenten.
}

Von Dr. Carl Arnold in Hannover.

Im deutschen Medicinalkalender für 1882 befindet sich eine vom Herausgeber desselben verfasste Abhandlung über procentige Arzneimischungen, die mich zu nachstehender Untersuchung veranlasste, und aus der ich Folgendes hervorhebe.

Im Allgemeinen versteht man unter procentigen Arzneimischungen solche, welche in 100 Theilen eine gewisse Menge eines Arzneimittels, der Basis, enthalten. Die Formel für eine solche Mischung aus 2 Stoffen $x$ und $y$ ist:

$$
1 / 100 \mathrm{x}+99 / 100 \mathrm{y}, 2 / 100 \mathrm{x}+98 / 100 \mathrm{y} \text { etc., }
$$

der Sprachgebrauch lässt jedoch auch zu

$$
1 \mathrm{x}+100 \mathrm{y}, 2 \mathrm{x}+100 \mathrm{y} \text { etc. }
$$

Wenn auch vielen Aerzten bei ihren Verordnungen das wahre Procent, die erste Formel, vorschweben mag - thatsächlich begegnet man bei solchen in Gewichtsmengen unter $100 \mathrm{~g}$. wenigstens fast nur der zweiten Formel. Im Allgemeinen aber verordnet der Arzt zur Zeit die Einzelgaben flüssiger Arzneien, die nach dem Gewichte bereitet sind, nach dem Maasse, d. h. Tropfenweise, Kaffee-, Esslöffelweise etc., also genau genommen in beinahe uncontrollirbarer Form, wenn man die Verschiedenheit der Tropfen und Löffel ins Auge fasst. Kommt aber schon bei innerlich zu nehmenden Arzneien der Unterschied zwischen Gewichts- und Volummenge in Betracht, so gewinnt dieses Verhältniss eine um so höhere Bedeutung bei denjenigen Arzneimischungen, welche zu Einspritzungen unter die Haut dienen sollen. Hier tritt die Verschiedenheit zwischen der nach dem Gewichte hergestellten und dem Maasse nach verbrauchten Flüssigkeit um so stärker hervor, als die bei der Mischung zweier Flüssigkeiten entstehende Aenderung des spec. Gewichtes und die bei der Lösung eines festen Arzneimittels in der Regel mit Verminderung des Volumens einhergehende Verdichtung sich bemerklich macht.

Ueber diese Verhältnisse zumal bei den bekanntesten und am meisten gebrauchten Injectionsflüssigkeiten fehlen nähere Angaben fast gänzlich.

Die in Grammen gewogene Flüssigkeit von unbekannten Volumen wird mit der Spritze dem Körper einverleibt. Die Spritze 
fasst aber bei bestimmter Temperatur nur von destillirtem Wasser von 1,0 spec. Gew. die gleiche Gewichts- und Maassmenge Wasser.

Je concentrirter eine dem Gewichte nach hergestellte Mischung von Fliissigkeiten oder eine Lösung ist und je mehr ihr spec. Gew. von 1,0 sich entfernt, um so mehr wird im Allgemeinen die Verschiedenheit zwischen Gewicht und Volumen, zwischen der Zahl der Gramme und der Zahl der Cubikcentimeter betragen.

Gewissenhafte Aerzte müssen daher die nach dem Gewicht bereitete Injectionsflüssigkeit nach dem Rauminhalte ihrer Spritzen messen, d. h. sehen, wie viele Füllungen der Spritze eine von ihnen verschriebene Injectionsflüssigkeit gibt, und darnach die Einzelgabe einer Einspritzung berechnen.

Wer sich nicht in dieser Weise helfen will, muss verlangen, dass seine Einspritzungsflüssigkeit wie die Normal- oder Maassflüssigkeit für die Volumetrie hergestellt, d. h. nicht gewogen, sondern durch Messung wie bei der Maass-Analyse bereitet werde, - eine Arbeit, welche allerdings zur Zeit noch gegen die Bestimmungen der Pharmacopöe verstösst, und in den verschiedenen Landes - Arzneitaxen nicht vorgesehen ist.

Waldenburg und Simon sagen in ihrer ArzneiverordnungsLehre 8. Aufl. pag. 87. „Sehr richtig ist eine genaue Dosirung der zu injicirenden Flüssigkeit. Hierzu gehört zunächst eine Kenntniss des Rauminhalts der in Gebrauch gezogenen Spritze. Die verschiedenen Spritzen variiren in dieser Beziehung innerhalb gewisser Grenzen, und es ist desshalb nothwendig, dass jeder Arzt den Inhalt seiner Spritze, bevor er dieselbe in Gebrauch zieht, genau abwiegt." Man nimmt diese Calibrirung am besten mit destillirtem Wasser von Zimmertemperatur vor, da die Lösung doch meist in der Umgebung des Patienten aufbewahrt wird. Ich habe nun die gebräuchlichsten zu subcutaner Injection verwendeten Medicamente, die von Waldenburg und Simon vorgeschriebene Dosirung als Ausgangspunkt nehmend, einer Bestimmung ihres spec. Gew. unterworfen. Die Bestimmungen wurden bei $20^{\circ} \mathrm{C}$. in einem mit genauem Thermometer versehenen Piknometer, sämmtliche Wägungen auf einer feinen chemischen Waage vorgenommen. Die als Basis dienenden Medicamente kamen lufttrocken und nach der Pharmacopöe auf ihre Reinheit geprüft in Anwendung. Die Lösungen wurden meist nach der gebräuchlicheren Formel $1 \mathrm{x}+100 \mathrm{y}$ 
dargestellt; es ist ja auch die Differenz der spec. Gew. von Lösungen der Formel $1 x+100 y$ und $1 / 100 x+99 / 100$ y eine ganz verschwindende.

Lösungen naeh der Formel $1 \mathrm{x}+100 \mathrm{y}=1 \%, 2 \mathrm{x}+100 \mathrm{y}=2 \%$ ete.

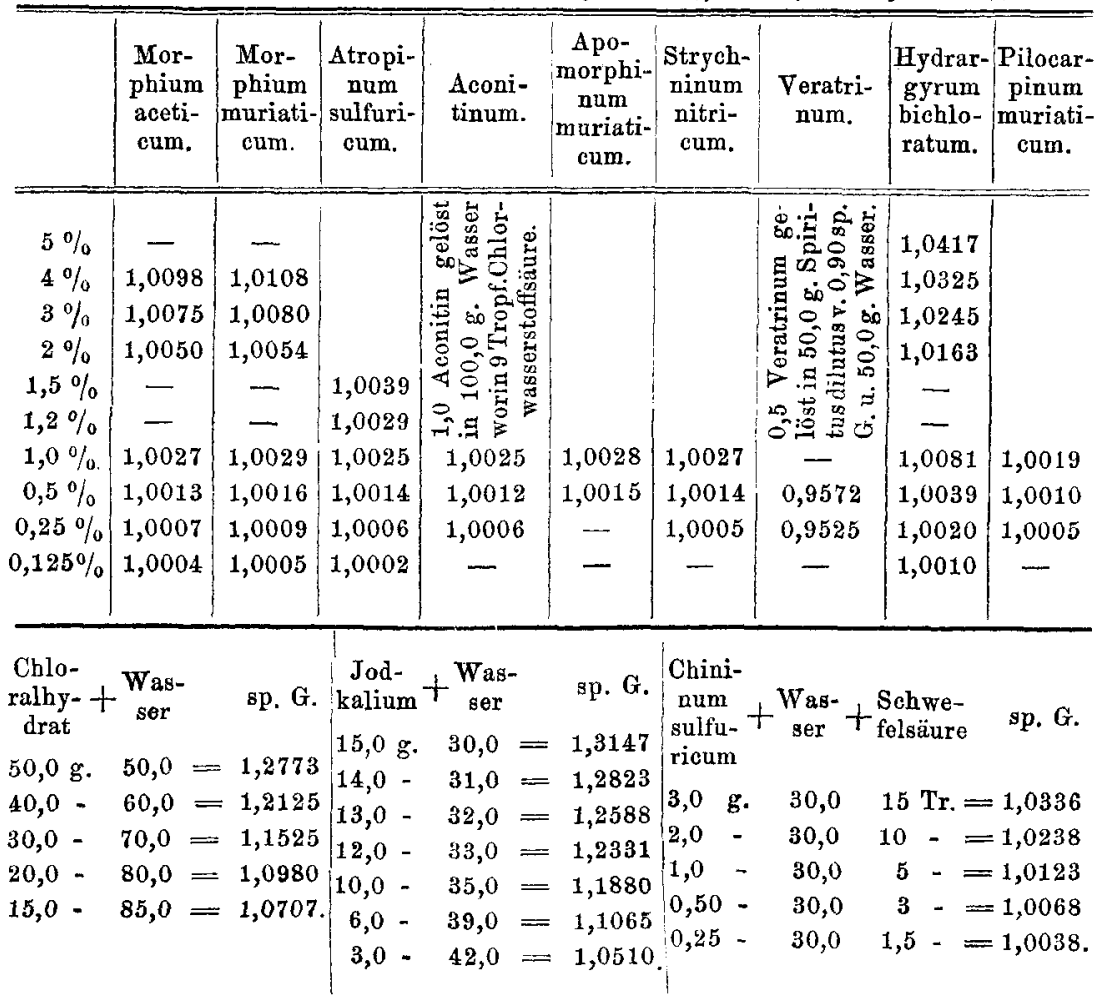

Wie aus vorstehender Tabelle zu ersehen, ist bei den heroischen Mitteln, selbst bei einer Dosirung wie sie zur subcutanen Injection nur in Ausnahmefällen zur Anwendung kommt, der Unterschied zwischen dem Volum und dem absoluten Gewichte ein so verschwindender, dass es in der Praxis vernachlässigt werden kann. Wenn von einer $4 \%$ Morphinlösung ein C.C. injicirt wird, so beträgt das Gewicht desselben 1,010 g. während der Patient bei Lösungen unserer Formel $1,04 \mathrm{~g}$. erhalten sollte. Nehmen wir eine nach der Formel $4 / 100 \mathrm{x}+{ }^{96 / 100} \mathrm{y}$ bereiteten Morphinlös!ng, so erhöht sich das spec. Gew. von 1,0108 auf 1,0110, der Patient erhält statt $1 \mathrm{C} . \mathrm{C} .=1 \mathrm{~g} .-1,011 \mathrm{~g} .=11 / 1000$ mehr. Es sind 
dies Differenzen, wie sie schon bei der Bereitung der Lösung stattfinden, da die zulässige Fehlergrenze bei den für Apotheken vorgeschriebenen Gewichten und Wagen viel grösser ist. Es unterliegt also keinem Bedenken, bei den Lösungen der Alkaloïde den Cubikcentimeter $=1 \mathrm{~g}$. anzunehmen. Bei Lösungen anderer zu subcutanem Gebrauche verwendeter Stoffe lässt sich durch einfache Rechnung aus dem gegebenen specifischen Gewichte das nöthige Volum ableiten. Eine Lösung von 5,0 g. Jodkalium in $10,0 \mathrm{~g}$. Wasser hat das spec. Gew. 1,3147, die 15,0 g. wiegende Lösung nimmt daber ein Volum ein von

$$
1,3147 \text { g. : } 15,0 \text { g. }=1 \text { C.C. : x C.C. }
$$

$$
\mathrm{x}=11,48 \text { C.C., woraus folgt, dass noch zuge- }
$$

fügt werden müssen 3,52 C.C. oder Gramme destillirtes Wasser, um eine Lösung zu erhalten, die in 15,00 C.C. - 5,0 g. Jodkalium enthält. Nennen wir $p$ das Gesammtgewicht der in der Tabelle für das betreffende spec. Gew. angefiuhrten Bestandtheile der Injection, $d$ das spec. Gow. der Injectionstlüssigkeit, $V$ die Zahl der gewünschten Ccm., auf welche die Lösung gebracht werden soll, so erhalten wir das zuzusetzende Wasser $X$ nach der Formel:

$V-\frac{p}{d}=X . \quad$ Nach obigem Beispiel, wo wir in $15 \mathrm{Ccm}$. der Lösung 5,0 g. Jodkalium verlangen, ist also der Lösung von $5,0 \mathrm{~g}$. Jodkalium in $10,0 \mathrm{~g}$. Wasser noch zuzusetzen:

$15-\frac{15}{1,3147}=3,52$ g. Wasser. Es wäre demnach zu verordnen. „Rp. g. 5,0 Kalii jodati solve in 13,50 g. Aqua destillata." Für den viel beschäftigten Arzt empfiehlt sich aber jedenfalis als kürzester Weg, dem Apotheker das gewünschte Volum der Injectionsflüssigkeit vorzuschreiben, unbekümmert darum, dass die Pharmacopöe das Messen von Flüssigkeiten nicht gestattet.

\section{Blechbüchsen von Zink zum Aufbewahren von Vegetabilien.}

Von Hofrath Dr. J. Ness ler in Carlsruhe.

Die durch Oxydation grau gewordene Oberfläche des Zinkbleches wiedersteht bekanntlich der Luft sehr gut. Auch die mit 